\title{
Therapeutic hypothermia and hypoxia-ischemia in the term-equivalent neonatal rat: characterization of a translational preclinical model
}

\author{
Shyama D. Patel', Leslie Pierce ${ }^{1,2}$, Amber Ciardiello', Alexandra Hutton', Samuel Paskewitz ${ }^{3}$, Eric Aronowitz ${ }^{4}$, \\ Henning U. Voss ${ }^{4,5}$, Holly Moore ${ }^{3,6}$ and Susan J. Vannucci ${ }^{1}$
}

BACKGROUND: Hypoxic-ischemic encephalopathy (HIE) is a major cause of morbidity in survivors. Therapeutic hypothermia $(\mathrm{TH})$ is the only available intervention, but the protection is incomplete. Preclinical studies of HIE/TH in the rodent have relied on the postnatal day (P) 7 rat whose brain approximates a 32-36 wk gestation infant, less relevant for these studies. We propose that $\mathrm{HIE}$ and $\mathrm{TH}$ in the term-equivalent $\mathrm{P} 10$ rat will be more translational.

METHODS: P10-11 rat pups were subjected to unilateral hypoxia-ischemia $(\mathrm{HI})$ and $4 \mathrm{~h}$ recovery in normothermic $(\mathrm{N})$ or hypothermic $(\mathrm{TH})$ conditions. Brain damage was assessed longitudinally at $24 \mathrm{~h}, 2 \mathrm{wk}$, and $12 \mathrm{wk}$. Motor function was assessed with the beam walk; recognition memory was measured by novel object recognition.

RESULTS: Neuroprotection with TH was apparent at 2 and $12 \mathrm{wk}$ in both moderately and severely damaged animals. TH improved motor function in moderate, but not severe, damage. Impaired object recognition occurred with severe damage with no evidence of protection of TH.

CONCLUSION: This adaptation of the immature rat model of $\mathrm{HI}$ provides a reproducible platform to further study HIE/TH in which individual animals are followed up longitudinally to provide a useful translational preclinical model.

$\mathbf{P}$ erinatal asphyxial brain damage resulting in hypoxicischemic encephalopathy (HIE) is a major cause of acute mortality and chronic neurological morbidity in infants and children $(1,2) ; 20-50 \%$ of infants with HIE die within the newborn period; up to $25 \%$ of survivors exhibit permanent neuropsychological handicaps. As there are no interventions to prevent perinatal asphyxia, the focus is on developing therapeutics to limit cerebral injury progression and promote normal brain growth and development (3).

Therapeutic hypothermia $(\mathrm{TH})$ has become standard therapy for full-term neonates with moderate-to-severe
HIE, with significant neuroprotection supported by multiple randomized clinical trials demonstrating reduced risk of death or neurologic disability at 18-24 mo of age (4-6). However, protection is incomplete, and long-term outcome is not well defined. TH may not improve outcome in neonates with very severe $\operatorname{HIE}(1,5)$. Additionally, at 6-7-year follow-up, there was no significant difference in IQ scores between the TH and control groups, suggesting a failure to support cognitive function (4). Thus, additional preclinical studies are needed to explore the benefits and limitations of $\mathrm{TH}$ and define adjunct therapies to enhance long-term outcome.

TH safety studies and clinical trials in the neonate were derived from experimental studies in newborn piglets (7) and fetal sheep (8), which demonstrated histopathological neuroprotection with moderate hypothermia. The immature rodent model of unilateral hypoxia-ischemia (HI) has been used extensively since a larger number of animals can be studied and longitudinal assessments and functional outcome can be measured (9). There have been multiple investigations into $\mathrm{TH}$, alone and with other therapies, in neonatal rats and mice, with quite variable results. Studies in the rat have used the P7 animal as originally described (10); however, the P7 rat brain more closely approximates a $32-36 \mathrm{wk}$ gestational age (GA) infant, potentially making it less relevant to the full-term newborn (11).

The purpose of this study was to define and characterize a reproducible, preclinical model of $\mathrm{HI}$ plus $\mathrm{TH}$ in the immature rat. We utilize rats of both sexes at P10-11, as representative of the term infant brain, and introduce a standard period of hypothermia following HI. Each pup is followed up longitudinally with magnetic resonance imaging (MRI) and behavioral analysis to assess short- and long-term effects. We demonstrate here significant reduction of brain damage and improved motor, but not object recognition, function with this TH paradigm. 


\section{RESULTS}

Hypothermic Protection Is Temperature Dependent

Following unilateral $\mathrm{HI}$, pups were recovered in either a normothermic $\left(35.5^{\circ} \mathrm{C}\right)$ or hypothermic $\left(30^{\circ} \mathrm{C}\right)$ environment for $4 \mathrm{~h}$. Hypothermic chamber temperature was chosen to achieve a target rectal temperature of $32{ }^{\circ} \mathrm{C}$; rectal temperatures were monitored hourly. Temperatures in normothermic (N) pups ranged from 36.5 to $38.1{ }^{\circ} \mathrm{C}$; hypothermic (TH) pups were $31-34^{\circ} \mathrm{C}$. In the initial studies, comparison of temperature with damage at $2 \mathrm{wk}$ showed that $\mathrm{TH}$ was most effective at $<33.0^{\circ} \mathrm{C}$; pups that remained $>33{ }^{\circ} \mathrm{C}$ were significantly more damaged ( $P \leq 0.01$; Supplementary Figure $S 1$ online). Pups that did not reach $33^{\circ} \mathrm{C}$ were excluded from subsequent analysis.

\section{Hypothermia Provides Long-Term Protection in Moderate and Severe Injury}

Preliminary studies assessed damage at $24 \mathrm{~h}$ in hematoxylin and eosin-stained sections; we did not observe a significant effect of cooling (Figure 1). However, cell death following $\mathrm{HI}$ can continue for days to months; long-term outcome in a developing brain represents a balance between cell death and growth of viable tissue. Subsequent experiments included longitudinal analysis, with MRI and behavior through $12 \mathrm{wk}$.

We performed 10 experiments of $\mathrm{HI}$ plus $\mathrm{TH}$; animals were evaluated with MRI at $2 \mathrm{wk}$. Damage was assessed with an ordinal damage score (DS) and calculation of regional volumes, as described in Methods (Supplementary Figure S2a,b online). There was a tight correlation between these two measures (Supplementary Figure S2c online). When all the data were considered together, there was a range of damage in both $\mathrm{N}$ and TH groups, with a trend toward lower DS with TH (Figure 2). On further examination, we observed that the experiments fell into three significantly different outcome groups based on the mean DS in the normothermic pups, reflecting mild $(n=3)$, moderate (N-M, $n=4)$, severe (N-S, $n=3$ ) injury (Figure 3 ).

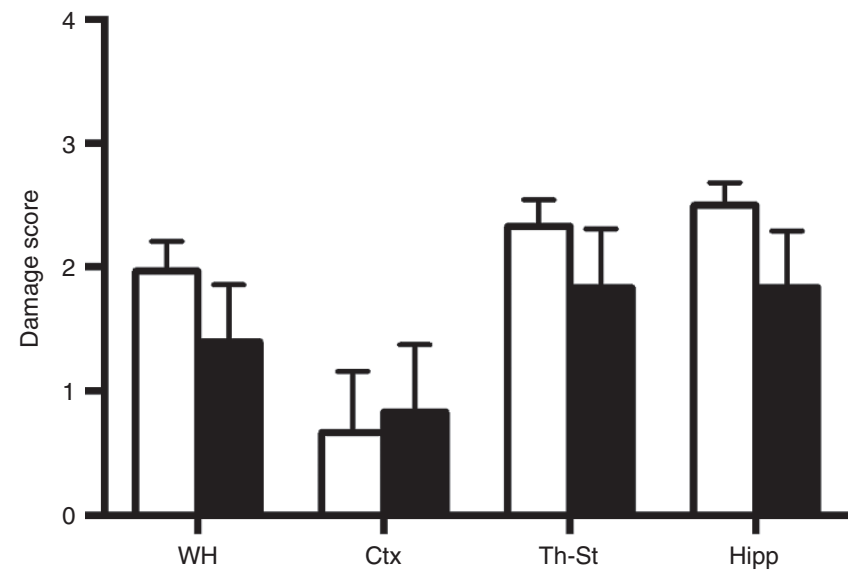

Figure 1. TH is not protective at 24 hours after HI. P10-11 rats were subjected to $\mathrm{HI}$ and recovered in normothermic (white bars) or hypothermic (black bars) chambers, $n=6 /$ group, and killed $24 \mathrm{~h}$ later. Hematoxylin and eosin-stained cryosections were analyzed for DS (ipsilateral) by two reviewers for whole hemisphere (WH), cortex (CTX), thalamus-striatum (Th-St), hippocampus (Hipp). HI, hypoxia-ischemia; $\mathrm{TH}$, therapeutic hypothermia.
TH did not significantly reduce damage in the mild group, and these animals were not followed up further. When moderate and severe experiments were analyzed separately, $\mathrm{TH}$ resulted

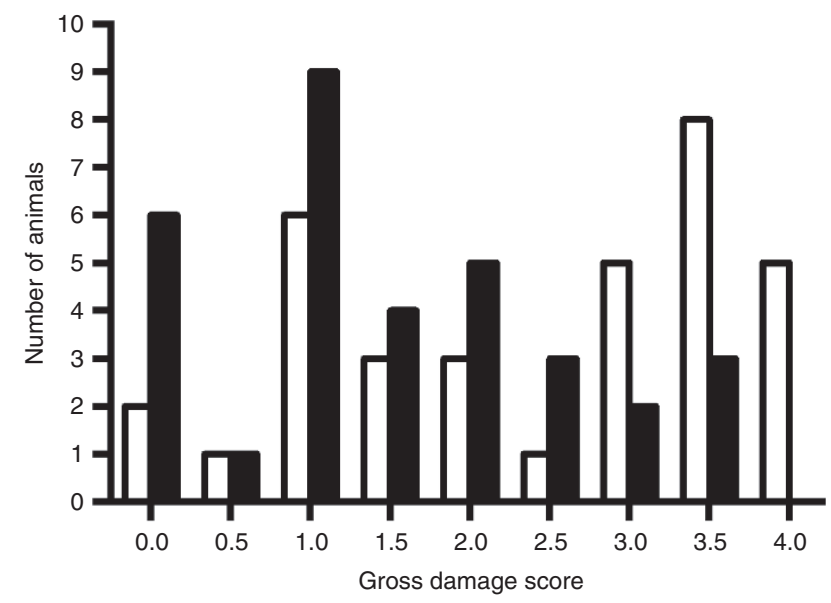

Figure 2. Effect of TH at 2 wk. DS was assessed in all animals from $T_{2}$ images demonstrating more animals with lower DS following TH (black bars) relative to normothermic (white bars) recovery. DS, damage score; $\mathrm{TH}$, therapeutic hypothermia.
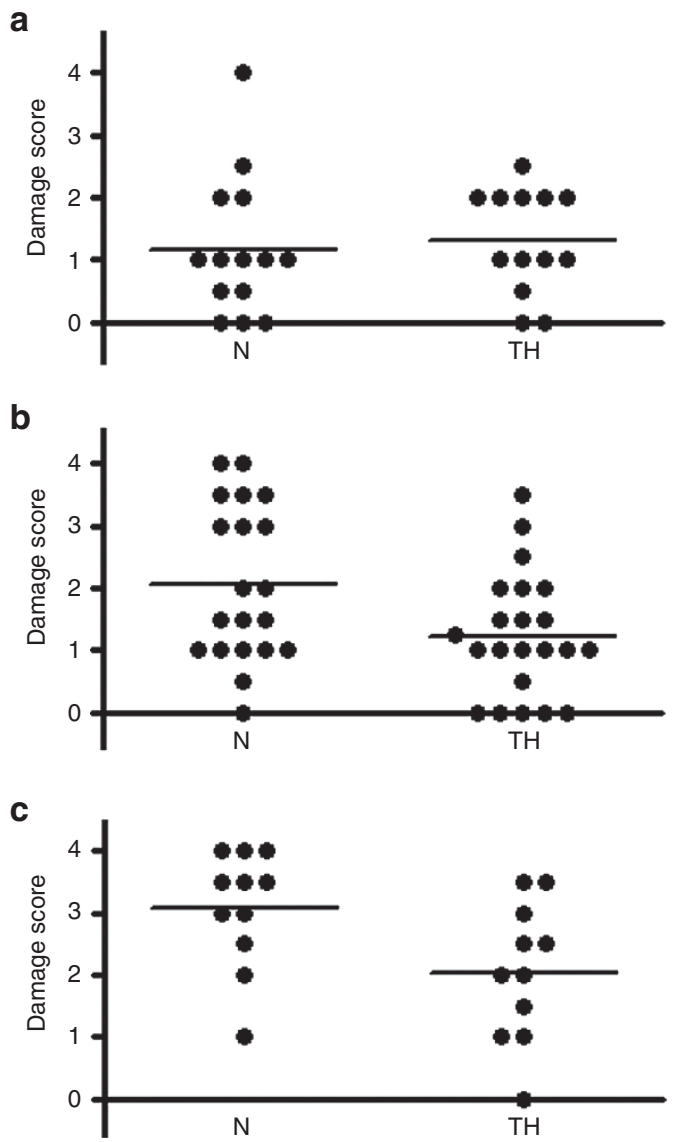

Figure 3. Effect of TH on mild, moderate, and severe damage. Experiments fell into three groups based on mean DS in normothermic (N) animals: (a) mild; (b) moderate; (c) severe $(P<0.01$, ANOVA). DS in the moderate and severe groups was reduced with $\mathrm{TH}(P<0.01$ for both, unpaired $t$-test). DS, damage score; $\mathrm{TH}$, therapeutic hypothermia. 
in a significant degree of protection in both groups (TH-M, TH-S, $P \leq 0.01$ ).

Regional volumes were calculated for ipsilateral and contralateral hemispheres (Figures 4a,b). TH was protective of neocortex and thalamus/striatum, but not hippocampus in both cohorts. Examination of individual ipsilateral/ contralateral ratios (Figure 4c) is a measure of extent of growth of the injured, relative to the contralateral, hemisphere; an abnormality in the contralateral hemisphere is determined by comparison with control. Contralateral volumes were not different from controls, except for a significant reduction in hippocampal volume in the severe cohort (Supplementary Figure S3 online), possibly indicating a heightened vulnerability of the hippocampus to HI that is resistant to TH. Figure 5 depicts the evolution vs. resolution of damage in 2 animals $(\mathrm{N}, \mathrm{TH})$, from the severe cohort: $24 \mathrm{~h}$ and $2 \mathrm{wk} \mathrm{T}_{2}$ MRI images, and histology of corresponding sections. Interestingly, despite little apparent difference at $24 \mathrm{~h}$, the injury in the $\mathrm{N}$ pup evolves into infarction and large cavitation at 2 and $12 \mathrm{wk}$; this process is reduced in the TH pup. Final DS derived from histological analysis of all brains at $12 \mathrm{wk}$ demonstrated both the evolution of injury and confirmed long-term protection with TH (Figure 6a,b). Calculated change in DS for each animal between 2 (Supplementary Figure S4 online) and 12 wk demonstrated minor evolution of damage in the N-M animals (Figure 6c), a
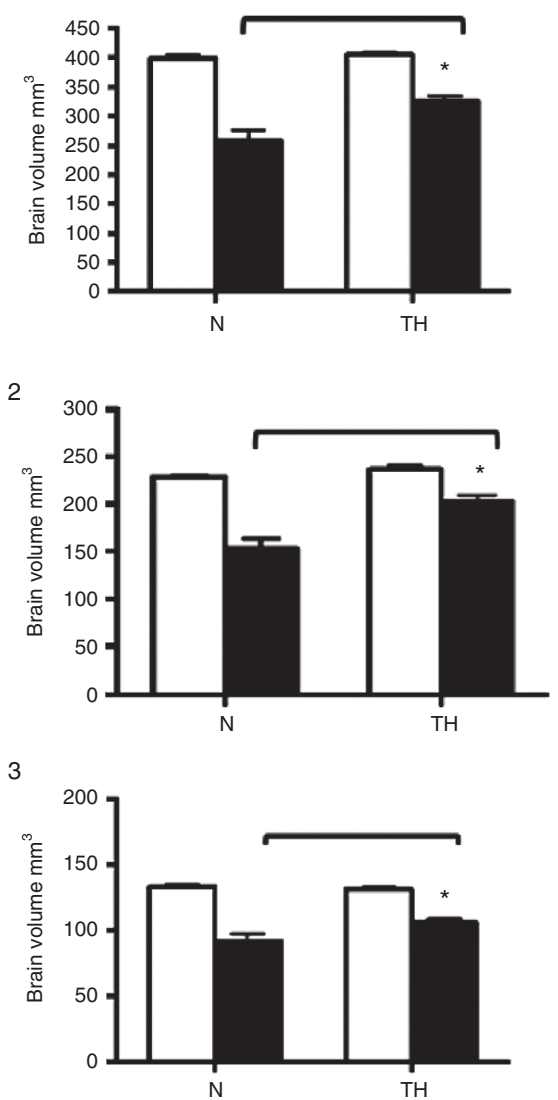

4

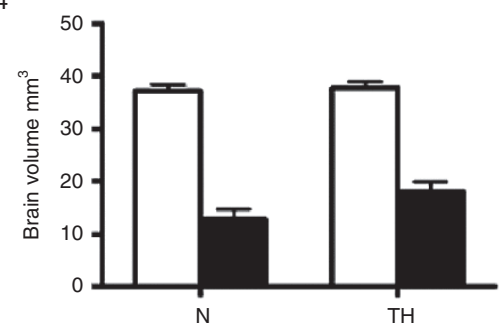

b

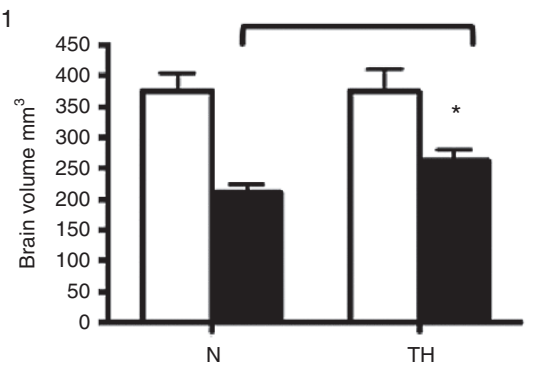

2

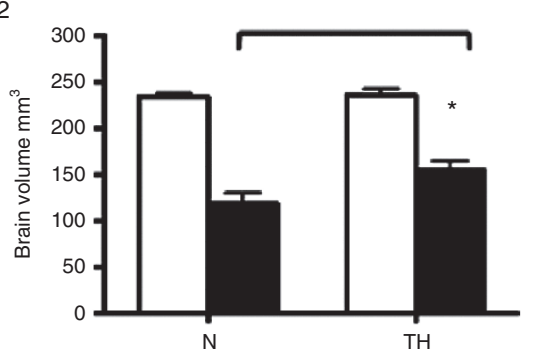

3

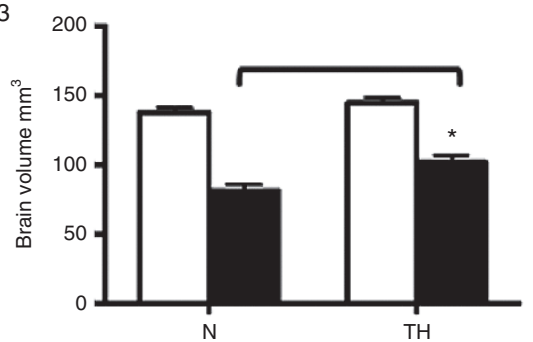

4

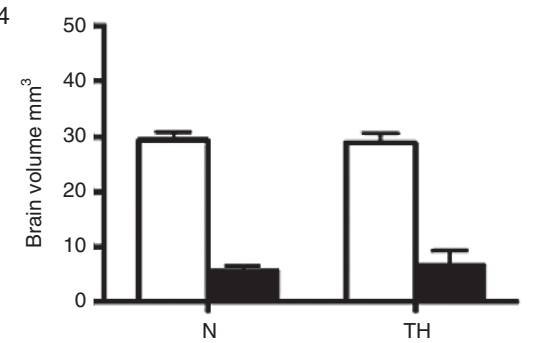

C

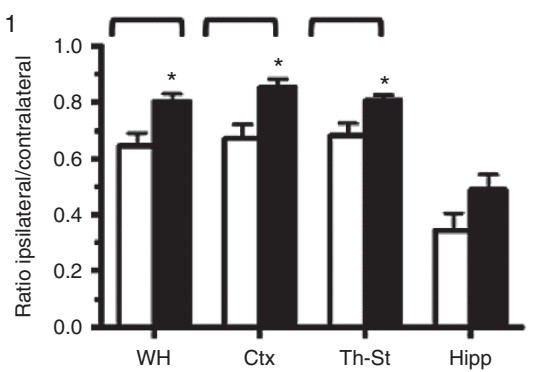

2

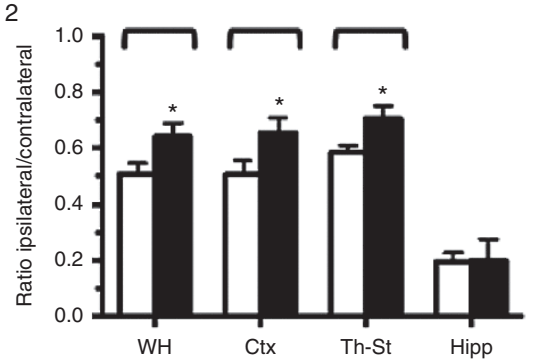

Figure 4. TH improves brain volumes at 2 wk except for hippocampus. Volumes of healthy brain tissue in contralateral (white bars) and ipsilateral (black bars) hemispheres were calculated from 2 wk MRI images as described in Methods. (a) Moderate, (b) severe; depicting volumes for: 1, whole hemisphere;

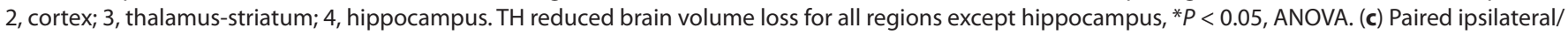
contralateral ratios, for normothermic (white bars) and TH (black bars) for: 1 , moderate; 2 , severe cohorts; ${ }^{*} P<0.05$. MRI, magnetic resonance imaging; TH, therapeutic hypothermia. 
a

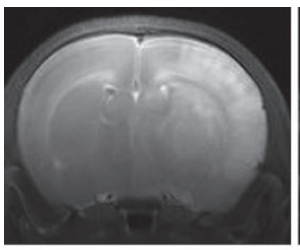

b

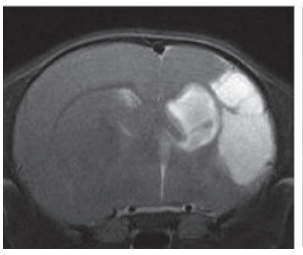

C

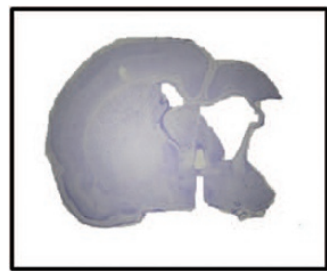

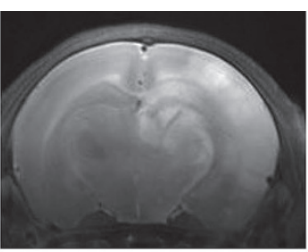
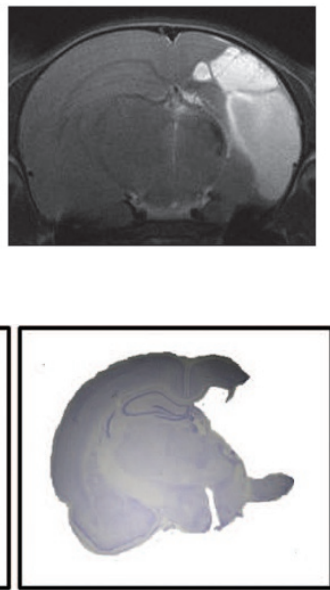

d

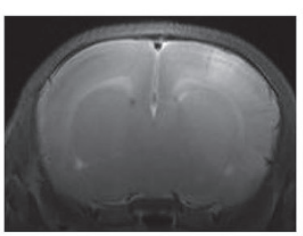

e

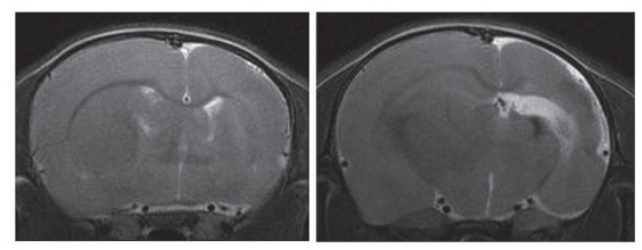

f

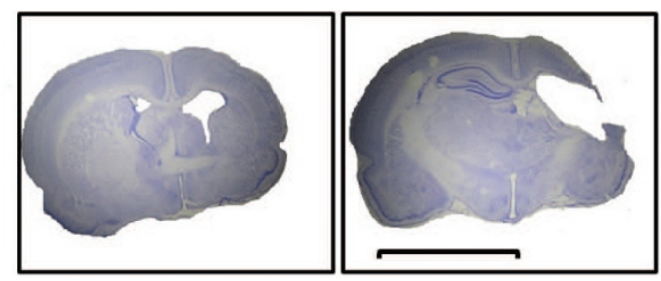

Figure 5. TH reduces progression of injury following $\mathrm{HI}$ : sequential $\mathrm{T}_{2} \mathrm{MRI}$ and histology. Representative MRI images obtained from $1 \mathrm{~N}(\mathbf{a}-\mathbf{c})$ and $1 \mathrm{TH}$ (d-f) pup at $24 \mathrm{~h}(\mathbf{a}, \mathbf{d})$ and $2 \mathrm{wk}(\mathbf{b}, \mathbf{e})$ are compared with images from final histology of each animal at $12 \mathrm{wk}(\mathbf{c}, \mathbf{f})$. Each panel depicts an anterior and posterior coronal section. Bar $=5 \mathrm{~mm}$. HI, hypoxia-ischemia; MRI, magnetic resonance imaging; $\mathrm{TH}$, therapeutic hypothermia.
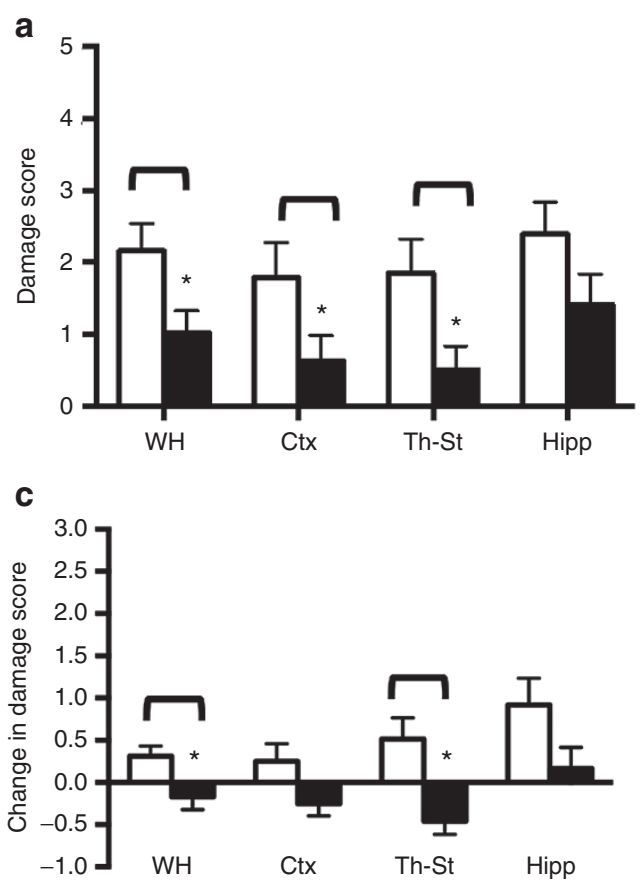

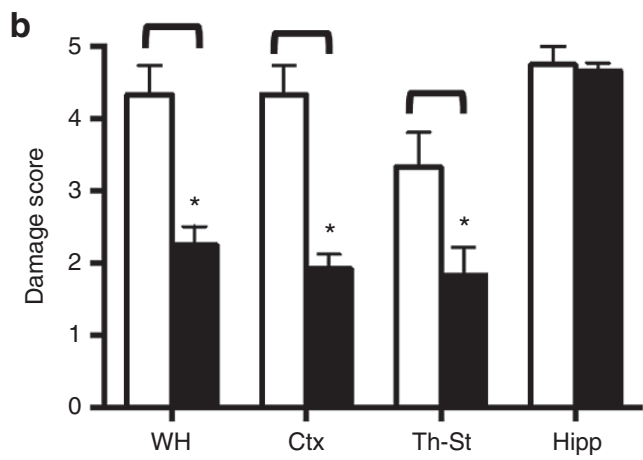

d

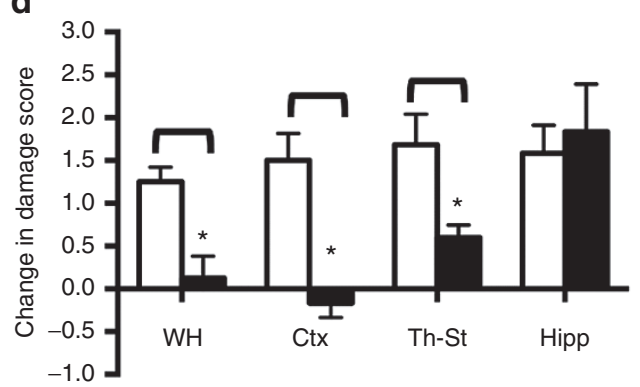

Figure 6. Effects of $\mathrm{HI}$ and TH on long term DS. Rats from (a) moderate and (b) severe cohorts were killed at 12 weeks post-HI. Cryosections were stained with cresyl violet and analyzed for hemispheric and regional DS as described. Change in DS between 2 and 12 wk was calculated for each animal in (c) moderate and (d) severe. White bars, normothermic; black bars, TH. ${ }^{*} P<0.05$, unpaired $t$-test. DS, damage score; HI, hypoxia-ischemia; TH, therapeutic hypothermia.

with more striking changes in the severe cohort (Figure 6d). In both cohorts, DS in the TH groups were either little changed or actually improved, except for the hippocampus.
In these studies, we did not observe a significant effect of gender on extent of damage or degree of protection by $\mathrm{TH}$ (Supplementary Figure S5 online). 
Hypothermia Protects Motor Learning and Dexterity but not Object Encoding and Recognition Following HIE

To characterize protection of sensorimotor function conferred by $\mathrm{TH}$, rats were tested as young adults (P50-70) on the beam walk test. This measurement of a rodent's ability to traverse an elevated beam is a robust test of locomotor control and is particularly sensitive to hindlimb deficits (12). Rats were tested on two beams of increasing difficulty. Successfully traversing the beam on at least 3 of 5 attempts was considered a "pass." The highest difficulty level passed differed as a function of group (Kruskal-Wallis test; $P<0.001$; Figure 7a). All of the $\mathrm{N}$ and $\mathrm{TH}$ animals in the moderate cohort, and all but one of the controls, passed both levels successfully. By contrast, none of the rats in the severe cohort passed both levels and several did not pass either (Figure 7a). Overall, the performance of this group was significantly worse than either controls or moderate groups (all $P$ values $<0.05$ ).

For rats that passed level 2, videos were analyzed for left (affected hindlimb) and right foot slips (Figure 7b). For left foot slips, the mixed ANOVA revealed an effect of group $(\mathrm{F}(2,19)=3.7 ; P<0.05)$ and an interaction between group and beam difficulty level $(\mathrm{F}(2,19)=4.7, P<0.05)$. Planned comparisons at each difficulty level revealed an effect of TH on motor control at the more difficult level $(\mathrm{F}(2,19)=5.9 P<0.05)$. N-M rats performed worse than both control and TH-M; the performance of the TH-M rats was significantly better than their noncooled littermates (Tukey honest significant difference; $P<0.05$ ) and not different from controls (Tukey honest significant difference $\mathrm{p}>0.4$ ). There were no group differences for right foot slips.

Temporal lobe-mediated object memory was investigated with novel object recognition (NOR), a behavioral test of encoding and memory that relies on the animal's innate exploratory behavior and requires no pretraining, and no added stressors $(13,14)$. NOR involves an initial exposure to two identical objects followed, after a delay, by presentation of one familiar object (from the previous sample phase) and one new object. Control animals spend significantly longer exploring the novel object; this is expressed by a discrimination index $\left(\mathrm{DI}_{\mathrm{NOR}}\right)$ calculated as the difference between time spent with the novel vs. familiar object as a percentage of total time.

The $\mathrm{DI}_{\mathrm{NOR}}$ differed across experimental groups $(\mathrm{F}(4,26)=3.1$; $P<0.05$; Figure 7c). As expected, naive controls spent more time investigating the novel than the familiar object; $\mathrm{DI}_{\mathrm{NOR}}$ significantly greater than 0 (one-sample $t$-test, $t(8)=3.1$; $P<0.05)$. Performance of moderate-HI animals $(\mathrm{N}-\mathrm{M}$ and TH-M) was not significantly different than controls (Student's $t$-tests; $P$ values $>0.15$ ) and indicated reliable recognition of the novel object $\left(\mathrm{DI}_{\mathrm{NOR}}>0\right.$; N-M: $t(4)=3.5$; TH-M: $t(5)=3.5$; $P$ values $<0.05)$. However, relative to controls, moderate-HI groups showed shorter object exploration times during the sample phase, with a trend toward increased exploration time in the TH-M group relative to N-M (Figure 7d). Object recognition was significantly impaired in the severe HIE groups, with N-S and TH-S rats performing no better than chance (Figure 7c).
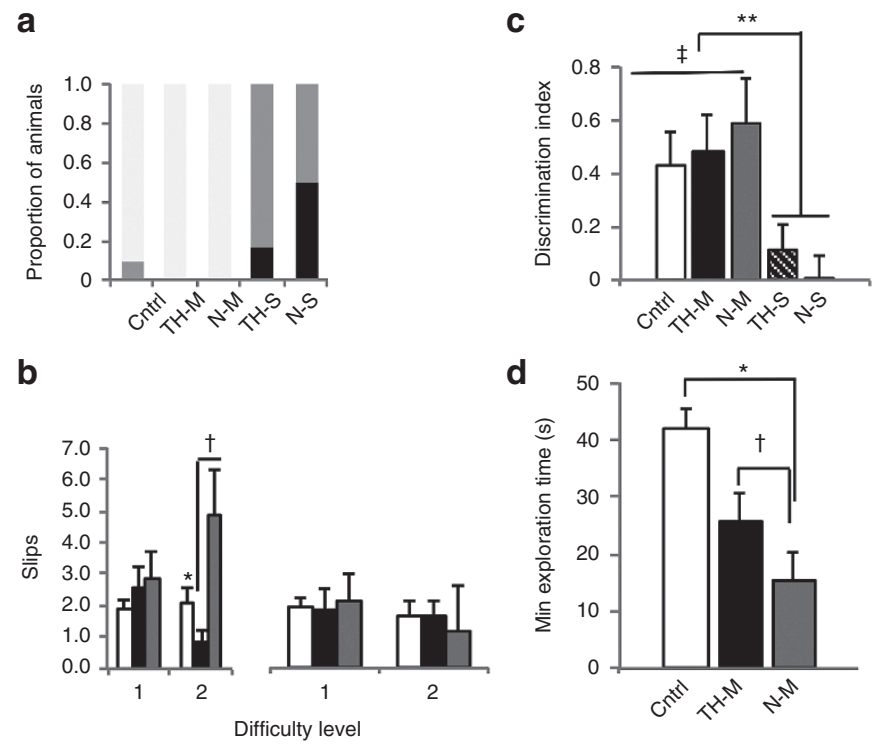

Figure 7. Motor and cognitive function in $\mathrm{TH}$ - and $\mathrm{N}$-treated $\mathrm{HI}$ rats. $\mathrm{TH}$ improves motor performance on the beam test. (a) Beam difficulty at first failure. Most rats in control (Cntrl) and moderate (-M) groups pass to level 2 (white bars); severe (-S) groups fail at (gray bars)/before (black bars) level 1. (b) Contralateral hindlimb slips (left panel) on the difficult beam are increased in rats with moderate HIE with normothermic recovery ( ${ }^{*} P<0.05, \mathrm{~N}-\mathrm{M}$, gray bars $>$ Cntrl, white bars); TH-M (black bars) rats are less impaired $\left({ }^{\dagger} P<0.05, \mathrm{TH}-\mathrm{M}<\mathrm{N}-\mathrm{M}\right)$. No difference in ipsilateral (right) hindlimb slips (right panel). Object encoding and recognition is impaired by HI. (c) Cntrl (white bars) and moderate HIE rats (TH-M, black bars; N-M, gray bars) show above-chance recognition of the novel object ( ${ }^{\ddagger} P<0.05$ $\left.\mathrm{DI}_{\mathrm{NOR}}>0\right)$; severe HIE rats do not $\left({ }^{* *} P<0.05\right.$, severe HIE:N-S (hatched bar) and TH-S (dark gray bar) vs. all other groups). (d) Object contact time for moderate HIE rats is less than for Cntrls (white bar) during the sample phase. There is a trend for TH (black bar) to mitigate this effect. ${ }^{*} P<0.05$, moderate HIE vs. Cntrl; ${ }^{\dagger} P<0.1, \mathrm{TH}-\mathrm{M}>\mathrm{N}-\mathrm{M}$ (gray bar). DS, damage score; $\mathrm{HIE}$, hypoxic-ischemic encephalopathy; $\mathrm{TH}$, therapeutic hypothermia.

\section{DISCUSSION}

In this study, we present a modification of our original model of $\mathrm{HI}$ in the P7 rat to more closely model the clinical situation of therapeutic hypothermia following birth asphyxia in the term infant. The major features of this modification are: (i) use of the P10-11 rat pup; (ii) temperature monitoring in all pups; (iii) 24-h MRI analysis to assess initial injury severity; (iv) subsequent $2 \mathrm{wk}$ MRI analysis for evaluation of injury progression; and (v) evaluation of sensorimotor and cognitive outcomes as adults prior to sacrifice and histopathology. We propose that this preclinical paradigm will provide a valuable platform to both further understand the benefits and limitations of hypothermia following $\mathrm{HI}$ and to evaluate potential adjunct therapeutics to improve outcome.

This model was originally developed in the P7 rat pup, which approximates the cerebral development of a $32-36 \mathrm{wk}$ gestation human infant (10). In the past $30 \mathrm{y}$, this model has come to be widely used as a preclinical model of HIE and was the paradigm in which the initial rodent studies of TH were conducted $(9,15,16)$. Subsequent studies investigated delayed hypothermia and combination therapies (17-20) with quite variable results and inconsistent protection with $\mathrm{TH}$. To more 
closely model the full-term infant brain we have chosen to investigate the effects of hypothermia in the P10-11 rat pup $(11,21,22)$. An important and potentially clinically relevant difference between the responses to $\mathrm{HI}$ at the different ages is the increased vulnerability of the hippocampus in the older rat pup, as previously described (23). The extreme vulnerability of the hippocampus to $\mathrm{HI}$ is replicated in the studies presented here, in both moderately and severely damaged animals. Further, the hippocampus was the only region that was not protected by $\mathrm{TH}$, as has been recently reported clinically (24). Severe HI injury resulted in nearly complete loss of the ipsilateral hippocampus, as well as a decrease in the contralateral hippocampal volume, which is a new observation in this model and will be investigated in future studies.

The rigorous control of temperature during hypothermia is vital to both the efficacy and reproducibility of the protection. Target temperature in clinical practice of TH in asphyxiated infants is $33.5-34.5^{\circ} \mathrm{C}(25,26)$, and most rodent studies aim to achieve a rectal temperature of $32-33^{\circ} \mathrm{C}$, as we did in the current study. Rectal temperature has been shown to correlate well with brain temperature in this model (16). Most studies monitor temperature in sentinel pups that are excluded from further analysis, based on the observation that the presence of the rectal temperature probe affects the outcome (27). We monitored rectal temperatures in all of the pups at hourly intervals and observed a range of post-HI temperatures in both normothermic and hypothermic animals. Importantly, we observed that those pups that "resisted" cooling and did not reach the target temperature of $<33^{\circ} \mathrm{C}$ demonstrated a variable degree of damage including the most severe, whereas none of the effectively cooled pups were severely damaged. The reason(s) for this variation in response are unknown; however, postischemic hyperthermia is frequently observed consistent with severe damage in preclinical studies and in asphyxiated infants (28). An inability to reach the target temperature during cooling might reflect a more severe insult at the outset, or some other variable in the animal. We employ a cutoff temperature of 33 ${ }^{\circ} \mathrm{C}$ as an exclusion criterion in these studies.

The animal-to-animal variability in this model is well known. Since the original publication in 1981, we, and others, have made numerous technical adjustments to minimize the variability, including controlling litter size, reduced time of anesthesia, prewarming, and prehypoxia. However, as in human infants, there is still a variable response to $\mathrm{HI}$, ranging from none to mild, moderate, or severe. The response to hypothermia is also variable with the greatest benefit observed in the context of moderate injury in both clinical trials and experimental studies. Thus, in order to evaluate the treatment effect, it is important to assess the severity of injury in each animal; MRI provides the tool to stratify experimental animals into mild, moderate, and severe injury groups. Of the experiments included in this study, three resulted in mild injury in normothermic pups with no added protection with TH (Figure 3); further analysis of the remaining pups showed that $4 \mathrm{~h}$ of $\mathrm{TH}$ immediately following $\mathrm{HI}$ in the P10-11 rat is protective in both moderate and severe injury.
An effective neuroprotective intervention must include long-term functional improvement, in addition to reduction in brain damage. Defects in locomotor and cognitive function are common in children with HIE and experimental animals following unilateral HI (29). A complete battery of behavioral tests will be needed to evaluate functional outcome. However, in this initial study, we used two simple tasks to evaluate sensorimotor function (beam walk) and recognition memory (NOR). Injury to the ipsilateral sensorimotor cortex is characteristic of this animal model, and the resulting motor impairments can be reliably detected by the beam walk test (12). The results of Figure 7 reinforce the importance of evaluating experimental animals in the context of severity of injury. Impairment in beam walking was significant in N-M and worse in N-S cohorts. TH cohorts showed improvement of locomotor function on the beam walk, relative to their corresponding normothermic animals. Although we did not fully evaluate improvement of performance with time, a retest of the severely damaged cohort did not improve their scores (data not shown). Future studies will initiate testing at a younger age to follow a potential trajectory of recovery of motor function and possible effects of $\mathrm{TH}$.

The NOR test was chosen for an initial evaluation of cognitive outcome as it relies on neuronal connections within the medial temporal lobe; object recognition has been shown to depend on temporal lobe circuits in both rodents and primates (3032 ). The test is suitable to rats across a developmental timeline from weaning to old age, requires no pretraining and does not require use of potential stressors (33). Based on the measurement of the $\mathrm{DI}_{\mathrm{NOR}}$ alone, it would appear that there was no effect of moderate HI damage in object recognition. However, HI animals did spend significantly less time with the objects in the familiarization/sample phase. Reduced exploration time has been reported before, both for neonatal HI (34) and traumatic brain injury in the adult rat (35). While preliminary, we note a trend for $\mathrm{TH}$ to normalize exploration time in the moderately damaged animals. Further testing will utilize an expanded NOR paradigm to tease apart relationships between object exploration and subsequent recognition, and the effects of HIE and TH. Severely damaged animals were unable to perform this task at all (Figure 7c). The apparent "sparing" of the moderate group might relate to extent of bilateral damage to hippocampal-perirhinal circuits. Recent studies on NOR highlight the importance of the perirhinal cortex $(31,32,36)$ and, although there is hippocampal damage in essentially all of the animals post-HI, the perirhinal cortex is more variably involved, especially in severe injury. It is noteworthy that while moderately damaged animals retained contralateral hippocampal volume, severely damaged animals did not, potentially compromising function in hippocampal-perirhinal circuits. It will be important to more fully characterize injury to hippocampal-perirhinal circuitry, and its relationship to NOR and other recognition memory tasks in this model over an extended timeline.

There are limitations to this study. MRI imaging is being increasingly utilized early in asphyxiated newborns to predict neurodevelopmental outcome (37-39). We have not yet 
analyzed our MRI $\mathrm{T}_{2}$ images to that extent, nor did we conduct a sufficient battery of neurodevelopmental tasks to fully assess outcome. However, this preclinical platform offers the opportunity to pursue such studies as well as include more advanced MRI imaging techniques to further define the trajectory of ongoing neurodegeneration or repair. Thus, we propose that this initial long-term study provides the basis for a useful, reproducible, and translationally relevant experimental paradigm to model the effects of therapeutic hypothermia following HIE in the term infant.

\section{METHODS}

\section{Animal Model of Unilateral HI Plus Hypothermia}

Timed pregnant Wistar rats (Charles River Laboratories, Wilmington, $\mathrm{MA}$ ) in groups of three were purchased at embryonic (E) day 15, housed individually, and allowed to deliver vaginally. On the day of birth, pups were randomized and reassigned to dams (10/litter, equal male and female).

Unilateral HI was induced in the right hemisphere at P10 according to our standard protocol $(10,40), 8 \% \mathrm{O}_{2} / \mathrm{bal} \mathrm{N}_{2}, 65 \mathrm{~min}$. During hypoxia, the temperature in the chamber was maintained at $34.5^{\circ} \mathrm{C}$ to achieve an average rectal temperature of $36-36.5^{\circ} \mathrm{C}$ (nesting temperature), monitored in two nonligated pups (Physitemp, Clifton, NJ). Pups were randomly assigned to either normothermic or hypothermic recovery for $4 \mathrm{~h}$. Normothermic recovery was in the same chamber as the hypoxia; hypothermic pups were placed in open jars in a water bath set at 28 to $30^{\circ} \mathrm{C}$ to maintain a target rectal temperature of $32^{\circ} \mathrm{C}$. Pups were rewarmed and placed back with the dam, weaned at P21, and handled every other day. Following MRI and behavioral analysis, animals were deeply anesthetized with a fatal dose of pentobarbital prior to perfusion-fixation with $4 \%$ paraformaldehyde. Brains were removed, cryoprotected, and $50 \mu \mathrm{m}$ sections were cut on a freezing microtome (Microm, Walldorf, Germany). All animal experiments were approved by the Weill Cornell Medical College IACUC.

\section{MRI Analysis}

Acquisition of images: $\mathrm{T}_{2}$-weighted images were acquired at $24 \mathrm{~h}$ and 2 -wk post-HI using a RÂRE sequence on a Bruker Biospec 7.0-T MR scanner with the following acquisition parameters: RARE factor 10, matrix $256 \times 256$, FOV $20 \times 20 \mathrm{~mm}$, slice thickness $1 \mathrm{~mm}, 17$ contiguous coronal sections at P11, and 20 contiguous coronal sections at $\mathrm{P} 25 \mathrm{TR}_{\text {eff }}=2,191.1 \mathrm{~ms}$ at P11 and $2,577.8 \mathrm{~ms}$ at P25, $\mathrm{TE}_{\text {eff }} 42.9 \mathrm{~ms}$. A $72 \mathrm{~mm}$ Bruker linear coil was used for excitation and a quadrature brain coil was used for signal reception. MRI images from Bregma 2.52 to -5.40 ( 8 images) were given a gross DS according to the ordinal scale: $0=$ no damage, $1=$ atrophy alone, $2=$ atrophy with ventriculomegaly, $3=10-25 \%$ infarct, and $4=25-100 \%$ infarct. Regions of interest (ROI) were traced (Supplementary Figure $\mathbf{2 b}$ online) using Image J software (NIH, Bethesda, MD). Regional volume measurements were calculated as the product of "intersection distance $(1 \mathrm{~mm}) \times$ area of normal tissue" for whole hemisphere, cortex, thalamus/striatum plus hypothalamus, and hippocampus.

\section{Histochemical Analysis}

Coronal brain sections from Bregma 2.52 to -5.40 ( 1 out of every sixth section) were mounted onto gelatin-coated slides. The sections were stained with a $1 \%$ cresyl violet solution (Sigma, St Louis, MO) and evaluated with light microscopy (Olympus, Tokyo, Japan). Using the same scoring system used for the MRI, overall and regional DSs were determined for each section.

\section{Behavioral Analysis}

The beam walking test was used to evaluate gross motor function at $6 \mathrm{wk}$ post-HI. Beams were $40 \mathrm{~cm}$ long, elevated $32 \mathrm{~cm}$ from the table surface. Level 1 beam was flat and $2.5 \mathrm{~cm}$ wide; level 2 beam was a cylindrical rod of $2.5 \mathrm{~cm}$ diameter. A dark box was at the end of the beam; rats instinctively advance into the box when placed on a beam. Rats were placed on the beam $10 \mathrm{~cm}$ from the box to learn the test. Then, each rat was placed $40 \mathrm{~cm}$ from the box and given five attempts to cross the beam. Videos were recorded and the fails, i.e., inability to cross, right and left foot slips were recorded by an investigator blinded to the treatment.

The novel object recognition test was performed at 8 -wk postHI. The day before the test, rats were allowed to explore the testing chamber, a polycarbonate box (dimensions $40 \times 40 \mathrm{~cm}$ ) and allowed to explore for $12 \mathrm{~min}$. The sample phase and recognition test were separated by a $6 \mathrm{~min}$. During the sample phase, two identical objects were placed near the corners of the box, and the rat was allowed to explore both objects for $5 \mathrm{~min}$. The rat was removed for $6 \mathrm{~min}$, wherein the box was cleaned with a chlorine dioxide liquid (Clidox; Pharmacal Research Laboratories, Waterbury, CT), and one of the familiar objects was replaced with a novel object of the same material but a different shape. The rat was returned to the box and allowed to explore for $2 \mathrm{~min}$. Any-Maze video tracking system software (Stoelting, Wooddale, IL) was used to record the time spent with novel and familiar objects. Object exploration was defined as the rat directing its nose toward the object at a distance of $<2 \mathrm{~cm}$. The videos were reviewed offline by two investigators blinded to the treatment. The $\mathrm{DI}_{\mathrm{NOR}}$ was calculated as ((time with novel object - time with familiar object) $) /($ total time spent with objects).

\section{Statistics}

All values were expressed as the mean \pm SEM. Effects of $\mathrm{HI}$ and $\mathrm{TH}$ were assessed with Kruskal-Wallis and ANOVA tests for noncontinuous and continuous variables, respectively. Omnibus tests were followed by planned pairwise comparisons. Post-hoc tests detecting an effect of TH were corrected for multiple comparisons with Tukey's honest significant difference method. Fisher's exact test and Dunnett's $t$ were also used for post-hoc analyses for the behavioral studies.

\section{SUPPLEMENTARY MATERIAL}

Supplementary material is linked to the online version of the paper at http://www.nature.com/pr

\section{ACKNOWLEDGMENTS}

We thank Jeffrey Perlman for support of these studies and helpful discussions.

\section{STATEMENT OF FINANCIAL SUPPORT}

This work was supported by a grant from the LeDucq Fondation, France (S.J.V.) and National Institutes of Health, USA (NIH)/R21NS083425 (S.J.V.). H.M. receives support from the Sidney R. Baer, Jr. Foundation and the New York State Office of Mental Health.

Disclosure: The authors have nothing to disclose.

\section{REFERENCES}

1. Wu YW, March WM, Croen LA, Grether JK, Escobar GJ, Newman TB. Perinatal stroke in children with motor impairment: a population-based study. Pediatrics 2004;114:612-9.

2. Vannucci RC. Hypoxic-ischemic encephalopathy. Am J Perinatol 2000;17:113-20.

3. Gonzalez FF, Ferriero DM. Therapeutics for neonatal brain injury. Pharmacol Ther 2008;120:43-53.

4. Shankaran S, Pappas A, McDonald SA, et al.; Eunice Kennedy Shriver NICHD Neonatal Research Network. Childhood outcomes after hypothermia for neonatal encephalopathy. N Engl J Med 2012;366:2085-92.

5. Gluckman PD, Wyatt JS, Azzopardi D, et al. Selective head cooling with mild systemic hypothermia after neonatal encephalopathy: multicentre randomised trial. Lancet 2005;365:663-70.

6. Azzopardi DV, Strohm B, Edwards AD, et al.; TOBY Study Group. Moderate hypothermia to treat perinatal asphyxial encephalopathy. $\mathrm{N}$ Engl J Med 2009;361:1349-58.

7. Thoresen M, Penrice J, Lorek A, et al. Mild hypothermia after severe transient hypoxia-ischemia ameliorates delayed cerebral energy failure in the newborn piglet. Pediatr Res 1995;37:667-70.

8. Gunn AJ, Gunn TR, de Haan HH, Williams CE, Gluckman PD. Dramatic neuronal rescue with prolonged selective head cooling after ischemia in fetal lambs. J Clin Invest 1997;99:248-56. 
9. Bona E, Hagberg H, Løberg EM, Bågenholm R, Thoresen M. Protective effects of moderate hypothermia after neonatal hypoxia-ischemia: shortand long-term outcome. Pediatr Res 1998;43:738-45.

10. Rice JE 3rd, Vannucci RC, Brierley JB. The influence of immaturity on hypoxic-ischemic brain damage in the rat. Ann Neurol 1981;9:131-41.

11. Patel SD, Pierce L, Ciardiello AJ, Vannucci SJ. Neonatal encephalopathy: pre-clinical studies in neuroprotection. Biochem Soc Trans 2014;42:564-8.

12. Goldstein LB. Model of recovery of locomotor ability after sensorimotor cortex injury in rats. ILAR J 2003;44:125-9.

13. Ennaceur A, Delacour J. A new one-trial test for neurobiological studies of memory in rats. 1: Behavioral data. Behav Brain Res 1988;31:47-59.

14. Antunes M, Biala G. The novel object recognition memory: neurobiology, test procedure, and its modifications. Cogn Process 2012;13:93-110.

15. Yager J, Towfighi J, Vannucci RC. Influence of mild hypothermia on hypoxic-ischemic brain damage in the immature rat. Pediatr Res 1993;34:525-9.

16. Thoresen M, Bågenholm R, Løberg EM, Apricena F, Kjellmer I. Posthypoxic cooling of neonatal rats provides protection against brain injury. Arch Dis Child Fetal Neonatal Ed 1996;74:F3-9.

17. Fang AY, Gonzalez FF, Sheldon RA, Ferriero DM. Effects of combination therapy using hypothermia and erythropoietin in a rat model of neonatal hypoxia-ischemia. Pediatr Res 2013;73:12-7.

18. Sabir H, Scull-Brown E, Liu X, Thoresen M. Immediate hypothermia is not neuroprotective after severe hypoxia-ischemia and is deleterious when delayed by 12 hours in neonatal rats. Stroke 2012;43:3364-70.

19. Liu Y, Barks JD, Xu G, Silverstein FS. Topiramate extends the therapeutic window for hypothermia-mediated neuroprotection after stroke in neonatal rats. Stroke 2004;35:1460-5.

20. Barks JD, Liu YQ, Shangguan Y, Silverstein FS. Phenobarbital augments hypothermic neuroprotection. Pediatr Res 2010;67:532-7.

21. Romijn HJ, Hofman MA, Gramsbergen A. At what age is the developing cerebral cortex of the rat comparable to that of the full-term newborn human baby? Early Hum Dev 1991;26:61-7.

22. Tucker AM, Aquilina K, Chakkarapani E, Hobbs CE, Thoresen M. Development of amplitude-integrated electroencephalography and interburst interval in the rat. Pediatr Res 2009;65:62-6.

23. Towfighi J, Mauger D, Vannucci RC, Vannucci SJ. Influence of age on the cerebral lesions in an immature rat model of cerebral hypoxia-ischemia: a light microscopic study. Brain Res Dev Brain Res 1997;100:149-60.

24. Kasdorf E, Engel M, Heier L, Perlman JM. Therapeutic hypothermia in neonates and selective hippocampal injury on diffusion-weighted magnetic resonance imaging. Pediatr Neurol 2014;51:104-8.

25. Azzopardi D, Strohm B, Edwards AD, et al.; Steering Group and TOBY Cooling Register participants. Treatment of asphyxiated newborns with moderate hypothermia in routine clinical practice: how cooling is managed in the UK outside a clinical trial. Arch Dis Child Fetal Neonatal Ed 2009;94:F260-4.
26. Papile LA, Baley JE, Benitz W, et al. Hypothermia and neonatal encephalopathy. Pediatrics 2014;133:1146-50.

27. Thoresen M, Bågenholm R, Løberg EM, Apriccna F. The stress of being restrained reduces brain damage after a hypoxic-ischaemic insult in the 7-day-old rat. Neuroreport 1996;7:481-4.

28. Laptook A, Tyson J, Shankaran S, et al.; National Institute of Child Health and Human Development Neonatal Research Network. Elevated temperature after hypoxic-ischemic encephalopathy: risk factor for adverse outcomes. Pediatrics 2008;122:491-9.

29. Schallert T, Fleming SM, Leasure JL, Tillerson JL, Bland ST. CNS plasticity and assessment of forelimb sensorimotor outcome in unilateral rat models of stroke, cortical ablation, parkinsonism and spinal cord injury. Neuropharmacology 2000;39:777-87.

30. Alvarado MC, Bachevalier J. Revisiting the maturation of medial temporal lobe memory functions in primates. Learn Mem 2000;7:244-56.

31. Barker GR, Warburton EC. When is the hippocampus involved in recognition memory? J Neurosci 2011;31:10721-31.

32. Winters BD, Forwood SE, Cowell RA, Saksida LM, Bussey TJ. Double dissociation between the effects of peri-postrhinal cortex and hippocampal lesions on tests of object recognition and spatial memory: heterogeneity of function within the temporal lobe. J Neurosci 2004;24:5901-8.

33. Reger ML, Hovda DA, Giza CC. Ontogeny of rat recognition memory measured by the novel object recognition task. Dev Psychobiol 2009;51: $672-8$.

34. Rojas JJ, Deniz BF, Miguel PM, et al. Effects of daily environmental enrichment on behavior and dendritic spine density in hippocampus following neonatal hypoxia-ischemia in the rat. Exp Neurol 2013;241:25-33.

35. Davis AR, Shear DA, Chen Z, Lu XC, Tortella FC. A comparison of two cognitive test paradigms in a penetrating brain injury model. J Neurosci Methods 2010;189:84-7.

36. Albasser MM, Davies M, Futter JE, Aggleton JP. Magnitude of the object recognition deficit associated with perirhinal cortex damage in rats: Effects of varying the lesion extent and the duration of the sample period. Behav Neurosci 2009;123:115-24.

37. Rutherford MA, Azzopardi D, Whitelaw A, et al. Mild hypothermia and the distribution of cerebral lesions in neonates with hypoxic-ischemic encephalopathy. Pediatrics 2005;116:1001-6.

38. Rutherford M, Pennock J, Schwieso J, Cowan F, Dubowitz L. Hypoxicischaemic encephalopathy: early and late magnetic resonance imaging findings in relation to outcome. Arch Dis Child Fetal Neonatal Ed 1996;75: F145-51.

39. Barkovich AJ, Hajnal BL, Vigneron D, et al. Prediction of neuromotor outcome in perinatal asphyxia: evaluation of MR scoring systems. AJNR Am J Neuroradiol 1998;19:143-9.

40. Vannucci SJ, Seaman LB, Vannucci RC. Effects of hypoxia-ischemia on GLUT1 and GLUT3 glucose transporters in immature rat brain. J Cereb Blood Flow Metab 1996;16:77-81. 